\title{
Analysis on Microbial Flora Changes During Processing and Storage of Spiced Goose Based on Pcr-Dgge Combined with Conventional Microbial Culture Methods
}

\author{
Xing $\mathrm{Li}^{1}$, Xiuqiong $\mathrm{Ou}^{1}$, Shaohong Jing ${ }^{1}$, Zhengze Zhong ${ }^{1}$, Lijun Bu${ }^{1}$, Huadong Xie ${ }^{1}$, Rui Li ${ }^{1 *}$, and Xiaochun Zhang ${ }^{1 *}$ \\ ${ }^{1}$ Chongqing Academy of Animal sciences, Chongqing, 402460, China and \\ Chongqing Engineering Research Center of Meat Quality Evaluation and Processing, Chongqing, 402460, China
}

\begin{abstract}
During the processing and storage process of spiced goose, samples were collected from raw goose meat (R0), spiced goose meat cooled for 2 hours (2h) and spiced goose meat stored for 1day (1d), 3 days (3d) and 5 days (5d). Microbial flora changes in spiced goose during the processing and storage process were analyzed by conventional microbial culture methods, polymerase chain reaction and denaturing gradient gel electrophoresis (PCR-DGGE). The results indicated that more abundant microbial diversity was determined in goose meat through PCR-DGGE technology than conventional microbial culture methods. According to the PCR-DGGE results, the dominant bacteria in R0 and $2 \mathrm{~h}$ were Aeromonas and Acinetobacter. On 1d, the dominant bacteria also included Weissella and Enterobacter, and on 3d, Aeromonas was no longer dominant. The dominant bacteria became Weissella, Enterococcus and Staphylococcus on 5d.
\end{abstract}

\section{Introduction}

Rongchang Spiced White Goose is a geographical indication product of Rongchang District in Chongqing. Based on statistics, the daily consumption of spiced goose in Rongchang District is 8000-10000. Due to its delicious flavor, spiced goose meat is popular, meanwhile the rich nutrients, high protein content and low fat content in spiced goose meat make it an ideal meat product[1]. Endogenous and exogenous microbial contamination occurs during slaughtering of geese, cleaning of raw goose meat and cooling process of spiced goose, resulting in the existence of certain microorganisms in the processed spiced goose products. The initial microorganisms will result in decay and deterioration of spiced goose during processing and storage and have the worry of food security. Studying changes in the microbial flora during the processing and storage of spiced goose is helpful to understand the pollution source of initial microorganisms and the microbial community of spiced goose meat, and provide a theoretical basis for prolonging the expiration date of spiced goose.

So far there has been little research on the microorganism of spiced goose. Lijun et al. studied the major pollution microorganisms and growth law in spiced goose during the production, transport, and sales process using conventional microbial culture methods[2]. At the same time studies on microbial flora changes in other domestic spiced meat products during processing and sale also were carried out using conventional microbial culture methods [3-4]. The results obtained from these methods are reliable and can be used to directly understand the dominant spoilage microorganisms in meat products. However, the results showed that more than $99 \%$ of the microorganisms in the natural environment were not able be cultured in the laboratory. Therefore, utilizing traditional culture methods to analyze the composition of microbial flora in meat products is not comprehensive and can cause the loss of some unculturable microorganisms[5-6]. Polymerase chain reactiondenaturing gradient gel electrophoresis (PCR-DGGE) is a molecular fingerprinting technique for investigating microbial community composition and their genetic relationships and is a powerful tool for bacterial classification and identification[7-10].that is able to efficiently, directly, quickly and comprehensively analyze microbial composition and community structure. This technique [11] can directly detect total bacterial DNA in a sample, determine the microbial species and abundance, as well as avoid the limitations of conventional microbial culture method in studying microorganisms that are hard to culture or uncultivable. However, this method can ' $t$ distinguish the distribution of living bacteria or nonliving bacteria, and can only qualitative analyses the microflora changes of meat products. Therefor some of studies investigate the microbial diversity of products based on PCR-DGGE combined with conventional microbial-culture methods [12-13]. In this study, the conventional microbial culture method and PCR-DGGE technology also were employed to investigate the microbial diversity of spiced goose during processing and storage, so as to understand the pollution source of initial microorganisms and the microbial community of spiced goose meat then

\footnotetext{
* Corresponding author:274842065@qq.com
} 
provide a theoretical basis for the preservation and to prolong the expiration date of spiced goose.

\section{Materials and methods}

\subsection{Materials and reagents}

A spiced goose processing enterprise and its sales outlets were selected as the sample source of this study. The raw goose and spiced goose products involved in the experiment were collected from the enterprise and the sales outlets.

The culture media used to detect the major pollution microorganisms during processing and storage process of spiced goose which listed in table 1 were: Plate count agar (PCA) for total aerobic bacteria, violet red bile agar (VRBA) for the Coliform, Baird-Parker agar and freezedried rabbit plasma for the Staphylococcus aureus, MRS medium, MC medium and MRS medium modified with mupirocin lithium salt for the Lactic acid bacteria, violet red bile glucose agar (VRBGA) for the Enterobacteriaceae, Bengal red agar for the mold and yeast, Pseudomonas CFC selective agar for the Pseudomonas and STAA agar for the Brochothrix thermosphact were purchased from Qingdao Hope BioTechnology Co.,Ltd. Agarose was brought from Oxoid (UK) and $2 \times$ Taq PCR MasterMix and DNA extraction kits were purchased from TIANGEN Biotech (Beijing) Co., Ltd. Proteinase K and urea were bought from Sigma (USA). Ammonium sulfate (APS), acrylamide (Acr) / methyl bis acrylamide (Bis) were from Bio-rad (USA). PCR primers were synthesized by BGI Tech Co., Ltd.

\subsection{Instruments and equipment}

Sterilization pot, Shanghai Boxun Industrial Co., Ltd. Medical Equipment Factory; GZX- GF101- II type electric heating constant temperature blast drying box, Shanghai Yuejin Medical Instrument Co., Ltd.; SW-CJ1FD type purification workbench, Suzhou Purification Equipment Co., Ltd.; BPC-150F biochemical incubator, Shanghai Yiheng Scientific Instrument Co., Ltd. MyCycler $^{\mathrm{TM}}$ Thermal Cycler PCR Instrument, GS 800 Gel Imaging Analysis System, D-code system and Horizontal Electrophoresis System, American Bio-rad Company; Vertical Low Temperature Refrigerator and Vertical Refrigerator, Qingdao Haier Group; High Speed Refrigerated Centrifuge, UK Thermo.

\subsection{Experimental methods}

\subsubsection{Sample processing}

Processing flow chart of spiced goose

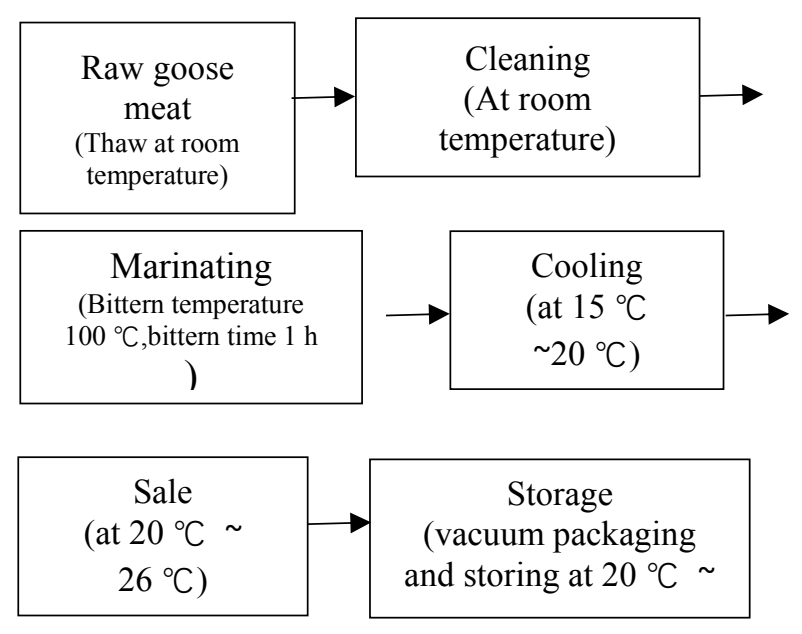

Clean raw goose meat, spiced goose meat cooled for $2 \mathrm{~h}$, as well as spiced goose meat vacuum-packed to store at $15{ }^{\circ} \mathrm{C}$ for $1 \mathrm{~d}, 3 \mathrm{~d}$ and $5 \mathrm{~d}$ from sales were collected to perform traditional microbial culture and PCR-DGGE analysis. Three independent geese of each group were sampled and tested.

\subsubsection{Determination of the major pollution microorganisms during processing and storage process of spiced goose by conventional microbial culture method}

$25 \mathrm{~g}$ samples were weighed under sterile conditions and pulverised in $225 \mathrm{ml}$ sterile saline. After vigorous shaking, $1 \mathrm{ml}$ sample was added into $9 \mathrm{ml}$ sterile saline to prepare $10^{-2}$ dilution. Based on the previous procedures, $10 \mathrm{x}$ serial diluted sample solutions were prepared. Three sample solutions of the appropriate dilution were selected to perform colony counting according to the requirements in the standard which listed in table 1 . The results obtained were statistically analyzed using excel.

\subsubsection{Determination of microbial flora changes during processing and storage of spiced goose by PCR-DGGE}

\section{(1) DNA extraction}

After each $9 \mathrm{~g}$ sample was cut into pieces, it was added to $91 \mathrm{~mL}$ sterile saline and homogenized for $10 \mathrm{~min}$. Subsequently, the microbial solution was centrifuged at $1000 \times \mathrm{g}$ for $3 \mathrm{~min}$ at $4^{\circ} \mathrm{C}$, and the supernatant was placed in another sterilized centrifuge tube to centrifuge at $8000 \times \mathrm{g}$ for $10 \mathrm{~min}$ at $4^{\circ} \mathrm{C}$. The pellet was collected and DNA was extracted using a DNA extraction kit (Beijing Tiangen) according to the manufacturer's instructions. After DNA was detected by $2.0 \%$ agarose and when the target strip appeared, it was stored in a refrigerator at $20^{\circ} \mathrm{C}$ for further use.

Table 1 Classification and culture conditions of microbial culture

(2) PCR amplification of bacterial $16 S$ rDNA fragments

Table 1 Classification and conditions of bacterial culture

\begin{tabular}{|c|c|c|c|}
\hline $\begin{array}{c}\text { Microbial } \\
\text { species }\end{array}$ & Medium & $\begin{array}{c}\text { Culture } \\
\text { condition }\end{array}$ & $\begin{array}{c}\text { Reference } \\
\text { standard }\end{array}$ \\
\hline
\end{tabular}




\begin{tabular}{|c|c|c|c|}
\hline $\begin{array}{l}\text { Total number } \\
\text { of colonies }\end{array}$ & PCA & $\begin{array}{c}36^{\circ} \mathrm{C} \pm 1^{\circ} \mathrm{C} \\
48 \mathrm{~h}\end{array}$ & GB 4789.2-2010 \\
\hline Coliform & VRBA & $\begin{array}{c}36^{\circ} \mathrm{C} \pm 1^{\circ} \mathrm{C} \\
24 \mathrm{~h}\end{array}$ & $\begin{array}{c}\text { GB } 4789.3-2010 \\
\text { Rule No. } 2\end{array}$ \\
\hline $\begin{array}{l}\text { Staphylococcu } \\
\text { s aureus }\end{array}$ & $\begin{array}{l}\text { Baird-Parker agar, } \\
\text { Freeze-dried rabbit } \\
\text { plasma }\end{array}$ & $\begin{array}{c}36^{\circ} \mathrm{C} \pm 1^{\circ} \mathrm{C} \\
48 \mathrm{~h}\end{array}$ & $\begin{array}{l}\text { GB 4789.10- } \\
2010 \\
\text { Rule No. } 2 \\
\end{array}$ \\
\hline $\begin{array}{l}\text { Lactic acid } \\
\text { bacteria }\end{array}$ & $\begin{array}{l}\text { MRS medium, MC } \\
\text { medium, MRS } \\
\text { medium modified } \\
\text { with mopiroxin } \\
\text { lithium salt }\end{array}$ & $\begin{array}{c}36^{\circ} \mathrm{C} \pm 1^{\circ} \mathrm{C} \\
48 \mathrm{~h}\end{array}$ & $\begin{array}{c}\text { GB } 4789.35- \\
2010\end{array}$ \\
\hline $\begin{array}{l}\text { Enterobacteria } \\
\text { ceae }\end{array}$ & VRBGA & $\begin{array}{c}36^{\circ} \mathrm{C} \pm 1^{\circ} \mathrm{C} \\
24 \mathrm{~h}\end{array}$ & $\begin{array}{c}\text { GB } 4789.41- \\
2016 \\
\text { Rule No. } 1 \\
\end{array}$ \\
\hline Mold, yeast & Bengal red agar & $\begin{array}{c}28^{\circ} \mathrm{C} \pm 1^{\circ} \mathrm{C} \\
5 \mathrm{~d}\end{array}$ & $\begin{array}{c}\text { GB 4789.15- } \\
2016 \\
\text { Rule No. } 1\end{array}$ \\
\hline Pseudomonas & CFC agar & $\begin{array}{c}25^{\circ} \mathrm{C} \pm 1^{\circ} \mathrm{C} \\
48 \mathrm{~h}\end{array}$ & $\mathrm{SN} / \mathrm{T} 4044-2014$ \\
\hline $\begin{array}{l}\text { Brochothrix } \\
\text { thermosphacta }\end{array}$ & STAA agar & $\begin{array}{c}30^{\circ} \mathrm{C} \pm 1^{\circ} \mathrm{C} \\
48 \mathrm{~h}\end{array}$ & ISO $13722-1996$ \\
\hline
\end{tabular}

HDA1-GC (5'-C G C C C G G G G C G C G C C C C GGGCGGGGCGGGGGCA C G G G G G A C T C C T A C G G G A G G C A G C A G T - 3') and HDA2( $5^{\prime}-\mathrm{G}$ T A T T A C C G C G G C T G C T G G $\mathrm{C}$ A $\mathrm{C}-3^{\prime}$ ) were used as the primers and the front end of the HDA1-GC primer had a GC cap structure. PCR reaction conditions $(25 \mu \mathrm{L}): 2 \times$ Taq MasterMix (CWBIO, Beijing) $12.5 \mu \mathrm{L}, 1 \mu \mathrm{L}$ of upstream and $1 \mu \mathrm{L}$ of downstream primers $(10 \mu \mathrm{mol} / \mathrm{L}), 1 \mu \mathrm{L}$ of template DNA, and adding $\mathrm{ddH}_{2} \mathrm{O}$ to achieve the final volume of $25 \mu \mathrm{L}$. A negative control group was designed. Reaction conditions were: $94{ }^{\circ} \mathrm{C}$ for $4 \mathrm{~min} ; 94^{\circ} \mathrm{C}$ for $30 \mathrm{~s}, 58{ }^{\circ} \mathrm{C}$ for $30 \mathrm{~s}, 72{ }^{\circ} \mathrm{C}$ for $2 \mathrm{~min}, 30$ cycles; $72{ }^{\circ} \mathrm{C}$ for $10 \mathrm{~min}$. The length, concentration of the PCR product fragment and the negative control were observed by $2.0 \%$ agarose gel electrophoresis. The PCR products were analyzed via denaturing gradient gel electrophoresis in $\mathrm{DCode}^{\mathrm{TM}}$ System electrophoresis apparatus, where the denaturation concentration ranged from $35 \%$ to $65 \%$. After electrophoresis for 5 minutes at $200 \mathrm{~V}$, electrophoresis was carried out at $60{ }^{\circ} \mathrm{C}$ and $100 \mathrm{~V}$ for $16 \mathrm{~h}$. The direction of electrophoresis was consistent with that of denaturation. After electrophoresis, staining was performed with silver nitrate staining, followed by imaging in a GS 800 gel imaging system (Bio-Rad, USA).

\section{(3) Band recovery and sequencing}

After PCR-DGGE staining, specific and non-specific DNA bands in each group were selected for DNA recovery and the DNA was recovered by polyacrylamide gel DNA recovery kit (Solarbio). The recovered fragments were free of GC clip primer to carry out PCR amplification according to the conditions in (2). When the PCR was complete, the DNA fragments were sequenced by Shanghai MAJORBIO Co. Ltd after visualizing the fragments on a $2.0 \%$ agarose gel.

(4) Data analysis
All data were sorted and imported into Excel for preliminary processing. Cluster analysis was performed using NTSYS-pc V2.10 (Exeter software, USA) and oneway ANOVA analysis was carried out by SPSS 19.0 (SPSS Inc., USA). A curve of obtained data was drawn using GraphPad Prism 6.0 (GraphPad Software Inc., USA). The resulting sequences were subjected to nucleic acid sequence homology analysis on the NCBI website (http: //blast.ncbi.nlm.nih.gov/ Blast.cgi).

\section{Results}

\subsection{Microbial flora changes of spiced goose during processing and storage analyzed by conventional microbial culture method}

As shown in Fig. 1, the total bacteria in the raw goose meat R0 was $4.66 \log \mathrm{CFU} / \mathrm{g}$. Since the temperature was kept at about $100{ }^{\circ} \mathrm{C}$ for $1 \mathrm{~h}$ during the stewing process and most of the microorganisms in the raw goose meat were killed, no colonies were detected in spiced goose meat after it was cooled for $2 \mathrm{~h}(2 \mathrm{~h})$. After $3 \mathrm{~d}$ of storage, the total number of colonies was $6.83 \log \mathrm{CFU} / \mathrm{g}$, which surpassed the maximum limitation $5 \mathrm{log}$ CFU/g in GB $2726-2016(2016)$. On the fifth day of storage, the total number of colonies reached $9.79 \log \mathrm{CFU} / \mathrm{g}$.

According to Fig. 1, the spoilage organisms in the raw goose meat (R0) mainly included Enterobacteriaceae, Pseudomonas, Lactic acid bacteria, molds and yeasts, and the numbers of these microorganisms had no significant difference. No colonies were detected in the spiced goose after it had been cooled for $2 \mathrm{~h}(2 \mathrm{~h})$. Only Lactic acid bacteria were detected in spiced goose meat stored for 1 $\mathrm{d}$ at $2.35 \log \mathrm{CFU} / \mathrm{g}$. On the third day of storage, the dominant organisms were Enterobacteriaceae, Lactic acid bacteria, Pseudomonas, Brochothrix thermosphacta, 
molds and yeast, and coliforms in order from most prevalent to least prevalent. On the fifth day of storage, the dominant organisms from most abundant to the least abundant were Enterobacteriaceae, Pseudomonas, Lactic acid bacteria, coliforms, Brochothrix thermosphacta, mold and yeast. During the storage of spiced goose, Lactic acid bacteria have always been the dominant bacteria, and due to the faster growth rate of Pseudomonas and Enterobacteriaceae, they finally developed into the dominant spoilage bacteria. The pathogen Staphylococcus aureus was not detected.

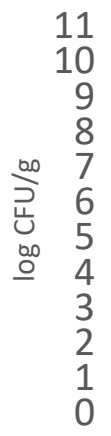
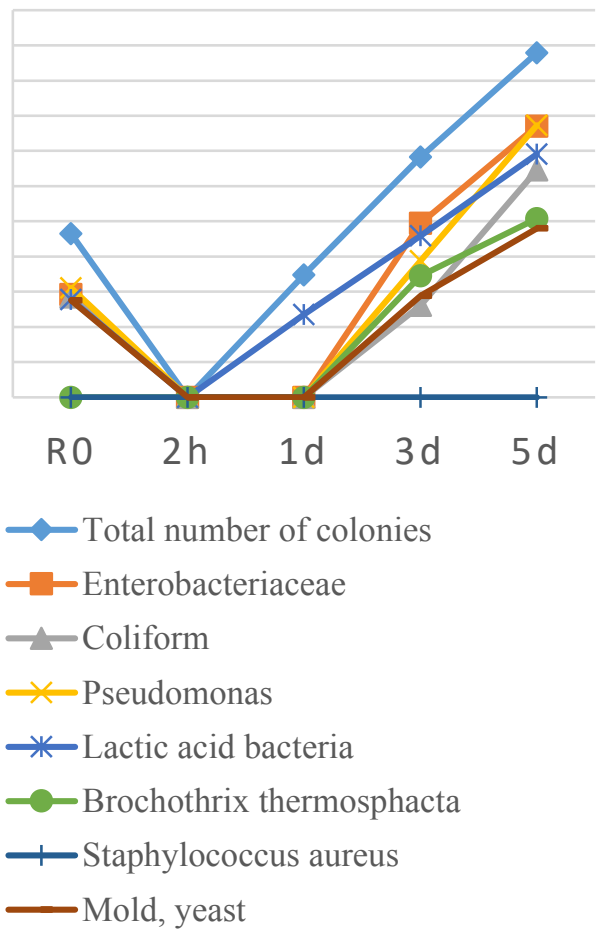

Fig.1. Microbial flora changes of spiced goose during processing and storage as determined by conventional microbial culture method

\subsection{Microbial flora changes of spiced goose during processing and storage process analyzed by PCR-DGGE technology}

\subsubsection{PCR-DGGE gels map sequencing results of spiced goose during processing and storage}

The PCR-DGGE detection results of bacteria in spiced goose during processing and storage are shown in Fig. 2. It can be seen that the repeatability among parallel samples was better, and the brightness of some bands was different. 37 main bands labeled in the profiles were recovered, cloned, and sequenced. As shown in Table 2, the sequence results were submitted to NCBI database for homology analysis. The results are shown in Table 1. Bands 1, 15, 27, 30, 31, 35 and 37 were Aeromonas sp., and strips 20, 24 belonged to Acinetobacter sp.; bands 33, 34, 36 were Enterobacter sp., and band 32 was Salmonella sp.; bands 3, 23, 28 were Streptococcus sp.; bands 4, 5, 7, 9, 10 and 13 belonged to Weissella sp.; band
8 was Lactococcus sp.; band 11 was Leuconostoc sp., and bands 14 and 26 were Enterococcus sp; band 17 was Carnobacterium sp.; band 2 was Oceanobacillus sp., and bands 18, 29 belonged to Staphylococcus sp. Bands 21, 22, 25 were of the genus Macrococcus; bands 6, 12, 16, 19 were uncultured bacteria.

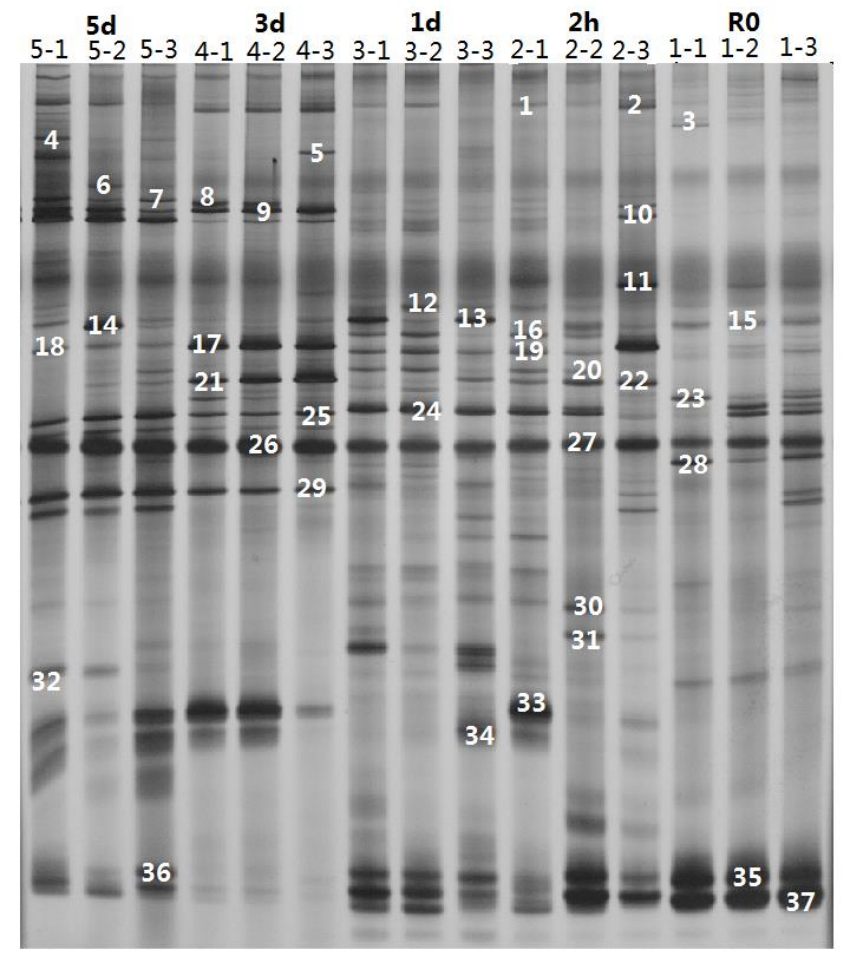

Fig. 2. PCR-DGGE Profiles of microbial flora in spiced goose during processing and storage

\subsubsection{Microbial flora changes of spiced goose during processing and storage process}

As can be seen from Fig. 2, the microbial flora in raw goose meat (R0) was composed of Aeromonas, Streptococcus, Acinetobacter and Macrococcus. Due to their brighter bands, Streptococcus, Acinetobacter and Aeromonas became the dominant bacteria. Aeromonas belongs to the Gram-negative bacteria[14] and since it was widely distributed in aquatic systems and soils, drinking water was a potential source of contamination. People can be infected mainly through consumption of water or food contaminated by the bacteria[15-16]After cooking at a high temperature for a long time, bands 3, 23, 28 in raw goose meat R0 disappeared and the streptococci were inactivated, as they were not detected during the subsequent storage of the spiced goose. The microbial flora in spiced goose meat after 2-hour cooling (2h) consisted of Aeromonas, Weissella, Acinetobacter, Macrococcus and Oceanobacillus, among which Aeromonas and Acinetobacter were the dominant bacteria. The positions of the bands in spiced goose stored for 1 day exhibited higher similarity to that after $2 \mathrm{~h}$, but the brightness of each band in the $1 \mathrm{~d}$ sample was higher. The dominant bacteria of $1 \mathrm{~d}$ included Weissella, Acinetobacter, Aeromonas and Enterobacter. When stored for 3 days, the Aeromonas bands disappeared. At this time, the dominant bacteria were Weissella, 
Carnobacterium, Enterococcus and Enterobacter. The bacterial composition in spiced goose meat stored for $5 \mathrm{~d}$ included Aeromonas, Weissella, Enterococcus,
Staphylococcus, Salmonella, Enterobacter, and the dominant bacteria were Weissella, Enterococcus and Staphylococcus.

Table 2. Gene sequence homology analysis results of microbial DGGE strips in spiced goose during processing and storage

\begin{tabular}{|c|c|c|c|}
\hline No. & Similar strains & $\underline{\text { ID }}$ & Similarity $(\%)$ \\
\hline 1 & Uncultured Aeromonadaceae bacterium & $\underline{\mathrm{LT} 695071}$ & $84 \%$ \\
\hline 2 & Oceanobacillus senegalensis strain Marseille-P3587 & NR_147387 & $95 \%$ \\
\hline 3 & Streptococcus dysgalactiae strain 14I & MF113285 & $97 \%$ \\
\hline 4 & Weissella cibaria strain AA10 & $\underline{\mathrm{JN} 851746}$ & $100 \%$ \\
\hline 5 & Weissella halotolerans & $\underline{\mathrm{LC} 064886}$ & $97 \%$ \\
\hline 6 & Uncultured bacterium isolate DGGE gel band bst_L05_01 & $\underline{\text { KU579293 }}$ & $98 \%$ \\
\hline 7 & Weissella cibaria strain AA10 & $\underline{\mathrm{JN} 851746}$ & $99 \%$ \\
\hline 8 & Lactococcus garvieae strain CIV.46 & $\underline{\text { MF628997 }}$ & $97 \%$ \\
\hline 9 & Weissella thailandensis strain CAU3107 & $\underline{\text { MF429421 }}$ & $99 \%$ \\
\hline 10 & Weissella confusa strain RCM3 & $\underline{\text { KT626390 }}$ & $100 \%$ \\
\hline 11 & Uncultured Leuconostoc sp. clone K-67-13-36 & $\underline{\mathrm{KJ} 804056}$ & $93 \%$ \\
\hline 12 & Uncultured bacterium clone 418 OIs2-E2 & $\underline{\mathrm{JN} 884204}$ & $97 \%$ \\
\hline 13 & Weissella paramesenteroides strain $\mathrm{RC} 2$ & $\underline{\mathrm{JN} 863617}$ & $100 \%$ \\
\hline 14 & Enterococcus faecium strain ZGP-Efa.8 & KM921921 & $99 \%$ \\
\hline 15 & Aeromonas veronii strain ASH07 & $\underline{\text { KU725740 }}$ & $100 \%$ \\
\hline 16 & Uncultured bacterium clone GXTJ5A301AC0K3 & $\underline{\text { KF323312 }}$ & $95 \%$ \\
\hline 17 & Carnobacterium sp. UST050418-652 & $\underline{\text { FJ596440 }}$ & $100 \%$ \\
\hline 18 & Staphylococcus kloosii strain A15_iii & KP728024 & $96 \%$ \\
\hline 19 & Uncultured bacterium clone SDSF_8F_e06 & $\underline{\mathrm{KC} 713055}$ & $97 \%$ \\
\hline 20 & Acinetobacter sp. 796 & $\underline{\text { KU670099 }}$ & $96 \%$ \\
\hline 21 & Macrococcus caseolyticus strain B03_06 & $\underline{\mathrm{KX} 681464}$ & $99 \%$ \\
\hline 22 & Uncultured Macrococcus sp. clone S5_E04 & $\underline{\mathrm{KP} 181835}$ & $85 \%$ \\
\hline 23 & Streptococcus parauberis strain HFTC0135 & $\underline{\text { MF574721 }}$ & $95 \%$ \\
\hline 24 & Acinetobacter sp. strain MSRC26 & $\underline{\text { MF924336 }}$ & $99 \%$ \\
\hline 25 & Macrococcus sp. IME1552 chromosome & $\underline{\mathrm{CP} 017156}$ & $95 \%$ \\
\hline 26 & Enterococcus ratti strain S3B & $\underline{\mathrm{KX} 830982}$ & $95 \%$ \\
\hline 27 & Aeromonas sp. NCCP-1748 & $\underline{\mathrm{LC} 270183}$ & $97 \%$ \\
\hline 28 & Streptococcus dysgalactiae strain 14I & $\underline{\text { MF113285 }}$ & $92 \%$ \\
\hline 29 & Staphylococcus epidermidis strain elva3 & $\underline{\text { KU376392.1 }}$ & $96 \%$ \\
\hline 30 & Aeromonas sp. strain G3 & $\underline{\mathrm{MF} 101687}$ & $100 \%$ \\
\hline 31 & Aeromonas sp. strain G3 & $\underline{\text { MF101687 }}$ & $100 \%$ \\
\hline 32 & Salmonella enterica subsp. arizonae & $\underline{\mathrm{AB} 273736}$ & $99 \%$ \\
\hline 33 & Enterobacter sp. strain GC4 & $\underline{\text { MF928411 }}$ & $100 \%$ \\
\hline 34 & Enterobacter sp. strain SA187 & $\underline{\mathrm{KX} 694407}$ & $99 \%$ \\
\hline 35 & Aeromonas sp. strain G3 & $\underline{\text { MF101687 }}$ & $100 \%$ \\
\hline 36 & Enterobacter sp. strain MSRC72 & $\underline{\text { MF924349 }}$ & $97 \%$ \\
\hline 37 & Aeromonas veronii strain AVZ01 & $\underline{\text { MF521598 }}$ & $100 \%$ \\
\hline
\end{tabular}

The dominant genus Streptococcus in raw goose meat was not detected after marinating and Aeromonas became the dominant bacteria in R0, $2 \mathrm{~h}$ and $1 \mathrm{~d}$ with the gradually declined content during storage process. Weissella started to be detected from $2 \mathrm{~h}$ and it was the dominant bacteria till the end of the storage. The result was consistent with that from the conventional microbial culture test. Through conventional microbial culture, Lactobacillus were 
detected at 1d of storage, which were the dominant bacteria from $1 \mathrm{~d}$ to $5 \mathrm{~d}$. Weissella is the dominant lactic acid bacteria in fermented foods such as white wine and soy sauce, which play a beneficial role in increasing the content of flavor substances and improving food safety during food fermentation[17-21]ome Weissella can synthesize and release a class of polypeptides or precursor polypeptides with antibacterial activities, so as to effectively inhibit the growth of spoilage microorganisms and pathogenic bacteria [22]. Weissella in spiced goose can not only increase the flavor of goose meat, but also inhibit the growth of microorganisms. At the end of storage, the number of pathogenic bacteria such as Enterococcus, Staphylococcus, Salmonella and other pathogenic bacteria gradually increased and become dominant spoilage bacteria. Similar results are achieved by Keping Ye,which investigate the changes of microbial community and counts of MAP pot-stewed duck wing (PSDW) under different packaging films and spices ratio during $15^{\circ} \mathrm{C}$ storage, PCR-DGGE analysis revealed that Staphylococcus equorum, Weissella sp., Leuconostoc mesenteroides became the dominating bacteria of PSDW at the end of storage[23].

\subsubsection{Analysis on similarity and diversity of microbial flora structure in spiced goose during processing and storage}

Based on the similarity of the PCR-DGGE profiles, a cluster analysis map was constructed to analyze the similarity of the microbial flora composition in spiced goose during processing and storage. The results are shown in Fig. 3. It can be seen that the similarity between two parallel samples were higher, while the lowest similarity was discovered in group $2 \mathrm{~h}(83 \%)$ among three samples at the same sampling time point. The similarity in the other groups was greater than $90 \%$. The community structure of the R0 group presented significant differences with other groups, indicating that most of the microorganisms in the raw goose were killed during marinating. The microorganisms of spiced goose during the storage process mainly came from the environment in which the spiced goose was cooled and the microorganisms carried by the operators. The similarity of the colony structure between $2 \mathrm{~h}$ and $1 \mathrm{~d}$ was $76 \%$, indicating that the microorganisms in the spiced goose had not multiplied. At $3 \mathrm{~d}$, the colony structure changed greatly compared with $2 \mathrm{~h}$ and $1 \mathrm{~d}$, suggesting that the microorganisms began to multiply at this time. At $5 \mathrm{~d}$, the spiced goose was severely spoiled, and the similarity of colony structure between $3 d$ and $5 d$ was $69 \%$.

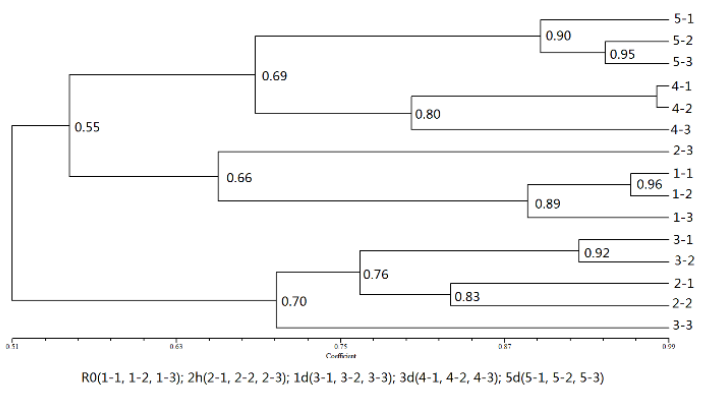

Fig. 3. Colony DGGE cluster analysis of spiced goose during processing and storage

The Shannon index $(\mathrm{H})$ was employed to evaluate the diversity of the microbial community. The larger the $\mathrm{H}$, the more the spices and numbers of microorganisms. The Simpson's index (D) indicated the degree of uniformity of the microbial distribution. The richness index (S) equates to the number of microbial species. According to Table 3, during the processing and storage of spiced goose, the $\mathrm{H}$ and $\mathrm{S}$ of microorganisms increased during storage. The species and quantity of microorganisms in raw goose meat (R0) were the lowest, and the highest types and quantities of microorganisms were achieved at $5 \mathrm{~d}$. The difference of $\mathrm{H}$ and $\mathrm{S}$ value was not significant between $2 \mathrm{~h}$ and $1 \mathrm{~d}$. After high temperature cooking, the microorganisms carried in the raw goose were killed, and the microorganisms in the environment during the cooling did not start to multiply in large quantities. From $1 \mathrm{~d}$ to $3 \mathrm{~d}$, the microorganisms began to multiply, and the number of previously dominant Aeromonas began to decrease remarkably due to inhibition by other dominant strains. At the same time, nutrients such as proteins in the goose were not degraded into available nutrients for microorganisms and the reproduction of other dominant bacteria had not reached the peak, and the $\mathrm{H}$ and $\mathrm{S}$ values were reduced at $3 \mathrm{~d}$. At $5 \mathrm{~d}$, the microorganisms multiplied and the number and types of microorganisms increased significantly, so the largest $\mathrm{H}$ and $\mathrm{S}$ values were achieved.

Table 3. Analysis on the microbial diversity of spiced goose during processing and storage

\begin{tabular}{|c|c|c|c|}
\hline Grouping & $\begin{array}{c}\text { Shannon } \\
\text { index (H) }\end{array}$ & $\begin{array}{c}\text { Simpson } \\
\text { index (D) }\end{array}$ & $\begin{array}{c}\text { Richness } \\
\text { index } \\
(S)\end{array}$ \\
\hline R0 & $2.98 \pm 0.08^{\mathrm{d}}$ & $0.75 \pm 0.02^{\mathrm{d}}$ & $19.67 \pm 1.53^{\mathrm{d}}$ \\
\hline $2 \mathrm{~h}$ & $3.28 \pm 0.10^{\mathrm{bc}}$ & $0.83 \pm 0.02^{\mathrm{bc}}$ & $26.67 \pm 2.52^{\mathrm{bc}}$ \\
\hline $1 \mathrm{~d}$ & $3.37 \pm 0.03^{\mathrm{ab}}$ & $0.85 \pm 0.01^{\mathrm{ab}}$ & $29.00 \pm 1.00^{\mathrm{ab}}$ \\
\hline $3 \mathrm{~d}$ & $3.22 \pm 0.04^{\mathrm{c}}$ & $0.81 \pm 0.01^{\mathrm{c}}$ & $25.00 \pm 1.00^{\mathrm{c}}$ \\
\hline $5 \mathrm{~d}$ & $3.44 \pm 0.07^{\mathrm{a}}$ & $0.87 \pm 0.02^{\mathrm{a}}$ & $31.33 \pm 2.08^{\mathrm{a}}$ \\
\hline
\end{tabular}

Note: The single factor sample analysis method was taken as the analysis method and the significant level was $0.05, \mathrm{n}=3$; the datas were expressed as mean $\pm \mathrm{SD}$, and the different letters indicated the significant difference.

\section{Conclusions}

In this paper, the conventional microbial culture method and PCR-DGGE technology were combined to analyze 
the microbial flora changes in spiced goose during processing and storage.

Through conventional microbial culture methods, the colonies in raw goose meat (R0) were $4.66 \log \mathrm{CFU} / \mathrm{g}$. The total number of colonies reached $9.79 \mathrm{log} \mathrm{CFU} / \mathrm{g}$ at $5 \mathrm{~d}$. The dominant spoilage microorganisms in R0 were mainly Enterobacteriaceae, Pseudomonas, Lactobacillus, mold and yeast. The dominant spoilage organisms at $5 \mathrm{~d}$ were Enterobacteriaceae, Lactobacillus and Pseudomonas.

Based on the results of PCR-DGGE detection, the number and types of microorganisms of spiced goose generally increased during processing and storage. The dominant bacteria of $\mathrm{R} 0$ and $2 \mathrm{~h}$ were Aeromonas and Acinetobacter, while at $1 \mathrm{~d}$ they were Weissella, Acinetobacter, Aeromonas and Enterobacter. Weissella, Carnobacterium, Enterococcus and Enterobacter became the dominant bacteria at $3 \mathrm{~d}$, and the dominant bacteria on 5d were Weissella, Enterococcus and Staphylococcus.

There was no microorganism was detected at $2 \mathrm{~h}$ using traditional microbial culture method, however Aeromonas and Staphylococcus were detected via PCR-DGGE technology. The reason is that PCR-DGGE technology can detect dead bacteria as well as live bacteria while traditional microbial culture method just detect live bacteria.

\section{References}

1. Jie Zh, Xinshu P, Sci Technol Food Ind 37 347-350 (2016)

2. Limei Y, Min Y, Jie W, Chunyang F, Xueson Zh, Food Res Dev 33 193-197 (2012)

3. ijun B, Tao X, Jun L, Baozhong L, Huadong X, Acad Period Farm Prod Proc 1 15-17 (2013)

4. Rui L, guojiao Zh, Hongxun W, China Brew 31 9395(2012)

5. Amann Ri, Ludwig W, Schleifer Kh, Microbiol Rev 59 143-169(1995)

6. Ranjard L, Poly F, Nazaret S, Res Microbiol 151 167-177(2000)
7. Pennacchia C, Ercolini D, Villani F, Food Microbiol 28 84-93 (2011)

8. Xiaofu D, Chongde W, Jun H, Food Res. Int. 62 1047-1053(2014)

9. Huibin Ch, Meiying W, Xiangzhi L, Food Microbiol 61 58-65(2017)

10. El Sheikha A F, Montet D, Crit Rev Food Sci Nutr 56 306-317 (2016)

11. Muyzer G, Waal E C D, Uitterlinden A G, Appl Environ Microbiol 59 695-700(1993)

12. Aifang L, Jing, Yunfang Q, Tian D, J Chinese Inst Food Sci Tech, 10 211-222(2018)

13. Xinlin ZH, Jing X, Yunfang Q, Yongji L, Danni Z, food sci, 37 271-277 (2016)

14. Dong Xiuzhu, Cai Miaoying (2001). Manual of Systematic Methods of Determinative Bacterial. (Beijing: Sci Press,2009)

15. Rusin P A, Rose J B, Haas C N, Rev Environ Contam Toxicol 152 57-83(1997)

16. Miyagi K, Hirai L, Sano, Japan. Environ Health Prev Med 21 287-294(2016)

17. Nolla-Salas J, Codina-Calero J, Vallés-Angulo S, Eur J Clin Microbiol Infect Dis 36 1393-1403(2017)

18. Qiaoyu L, Fang F, Guocheng D, Food Ferment Ind 43 241-247 (2017)

19. Fusco V, Quero G M, Cho G S, 6 155-176(2016)

20. Duskova M, Kamenik J, Karpiskova R, Acta Veter Brun 82 237-241(2013)

21. Oh S J, Shin N R, Hyun D W, Int J Syst Evol Microbiol 6 2951-2956(2013)

22. Qiaoyu L, Detection of characteristics of Weissella strains and their contents in soy sauce moromi mash during soy sauce fermentation( Jiangnan University, 2018)

23. Keping Y,Jian J, Yuefan W, Yifan H, Mei L, Jia L, Chengxiang G, J Food Sci Technol, 5 10731079(2017) 